\title{
Management of vertigo: from evidence to clinical practice
}

\author{
Paola Gnerre, ${ }^{1}$ Carlotta Casati, ${ }^{2}$ Mariella Frualdo, ${ }^{3}$ Maurizio Cavalleri, ${ }^{4}$ Sara Guizzetti ${ }^{5}$ \\ ${ }^{1}$ Medicina Interna, Ospedale San Paolo, Savona; ${ }^{2}$ Medicina Interna, AO Careggi, Firenze; ${ }^{3}$ Clinica Medica 1 , Policlinico A. \\ Murri, Bari; ${ }^{4}$ Medicina Interna, Ospedale di Sestri Levante, Sestri Levante (GE); ${ }^{5}$ Clinica di Medicina Interna I, IRCCS AOU \\ San Martino-IST Genova, Italy
}

\begin{abstract}
Vertigo is a symptom, not a diagnosis and effective diagnosis and management begin with understanding what this symptom may represent. Thus the presence of postural insility includes a differential diagnosis for otologic, neurologic and other medical causes. The objective of this paper is to provide evidence-based recommendations for the proper management of vertigo by multi-parametric analysis of the guidelines available to date.
\end{abstract}

\section{Introduction}

Although an appropriate and univocal definition of vertigo is often difficult, generally the term vertigo (from Latin relate) indicates a clinical symptomatology characterized by an unpleasant feeling of displacement of the body (subjective vertigo) or of the surrounding environment (objective vertigo). ${ }^{1}$ Vertigo is a symptom, not a diagnosis and effective diagnosis and management begin with understanding what this symptom may represents. Vertigo may be a component of a patient's dizziness (Table 1). ${ }^{1}$ Dizziness is nonspecific and it may result from a disorder of almost any organ system. ${ }^{1}$ Thus the presence of postural instability includes a differential diagnosis for otologic, neurologic and other medical causes. Vascular disorders cause pre-syncope dizziness as a result of cardiac dysrhythmia, orthostatic hypotension, medication effects, or another cause. Vestibular causes of dizziness may be due to peripheral lesions that affect the

\footnotetext{
Correspondence: Paola Gnerre, Medicina Interna, Ospedale San Paolo, via Genova 30, 17100 Savona, Italy. Tel.: +30.019.8404082.

E-mail: pgnerre@yahoo.it

Key words: Vertigo; subjective vertigo; objective vertigo; dizziness.

Received for publication: 4 October 2013.

Revision received: 16 June 2014.

Accepted for publication: 16 June 2014.

This work is licensed under a Creative Commons Attribution NonCommercial 3.0 License (CC BY-NC 3.0).

CCopyright P. Gnerre et al., 2015

Licensee PAGEPress, Italy

Italian Journal of Medicine 2015; 9:180-192

doi:10.4081/itjm.2015.437
}

labyrinths or vestibular nerves or due to involvement of the central vestibular pathways. ${ }^{2}$ Vestibular cause may be paroxysmal or due to a fixed unilateral or bilateral vestibular deficit. Therefore a careful history and examination often provide enough information to answer these questions and determine whether additional studies or referral to a specialist are necessary.

\section{Epidemiology}

Dizziness accounts for 5.6 million clinic visits in the United States per year. Therefore vertigo is a common symptom and affects $20 \%$ to $30 \%$ of the general population. ${ }^{3}$ The annual incidence is approximately $3.1 \%$, while the prevalence is $22.9 \% .{ }^{4}$ Vertigo or dizziness also accounted for $4 \%$ of presentations to emergency department in the years 1995 to $2004 .^{5}$ The dizziness is one of the causes of lack of work due to illness $(0.9 \%$ in women and $0.7 \%$ in men) and a major cause of disability pension ( $23 \%$ women and $24 \%$ men). ${ }^{6}$ Peripheral disorders account for $24-43 \%$ of Emergency Department (ED) dizzy patients ${ }^{7,8}$ and main cause is vestibular vertigo. The most common vestibular disorders seen in the ED are benign paroxysmal positional vertigo (BPPV) and acute peripheral vestibulopathy (APV) i.e., vestibular neuritis and labyrinthitis. ${ }^{9}$

\section{Causes of vertigo}

Vertigo can also be a sense of swaying or tilting. Some people perceive self-motion (subjective vertigo) whereas others perceive motion of the environment (objective vertigo). Vertigo arises because of asymmetry in the vestibular system due to damage or to dysfunction of the labyrinth, vestibular nerve, or central vestibular structures in the brainstem. Therefore conventionally causes of vertigo are separated into central or peripheral origins (Table 2). ${ }^{10,11}$ The central nervous system 
receives signals from both the right and left labyrinths and compares these signals with one another. When the head is still, tonic discharges in both vestibular afferents are exactly balanced. During motion, the right and left labyrinths are alternately excited and inhibited, leading to a left-right difference in eighth nerve activity, which is recognized as motion. The spurious left-right differences that result from an acute unilateral peripheral vestibular disorder are also interpreted by the central nervous system as motion or vertigo. ${ }^{10}$ Vertigo caused by problems with the inner ear or vestibular system, which is composed of the semicircular canals, the otolith (utricle and saccule), and the vestibular nerve is called peripheral, otologic or vestibular vertigo. The most common cause of peripheral vertigo is BPPV, Ménière's disease (MD), superior canal dehiscence syndrome, labyrinthitis and visual vertigo. ${ }^{11}$ Any cause of inflammation such as common cold, influenza, and bacterial infections may cause transient vertigo if it involves the inner ear, as well as chemical insults (e.g., aminoglycosides) or physical trauma (e.g., skull fractures). Motion sickness is sometimes classified as a cause of peripheral vertigo. Patients with peripheral vertigo typically present with mild to moderate imbalance, nausea, vomiting, hearing loss, tinnitus, fullness, and pain in the ear. In addition, lesions of the internal auditory canal may be associated with ipsilateral facial weakness. Central vertigo is vertigo due to a disease originating from the central nervous system (CNS). ${ }^{12}$ Central vertigo may be caused by hemorrhagic or ischemic insults to the cerebellum, the vestibular nuclei, and their connections within the brain stem. Other causes include CNS tumors, infection, trauma, and multiple sclerosis. Table $3^{11}$ shows the main features of central and peripheral vertigo.

\section{Peripheral disorders}

\section{Benign paroxysmal positional vertigo}

\section{Introduction}

BPPV is a common clinical disorder characterized by brief recurrent spells of vertigo often brought about by certain head position changes as may occur with looking up, turning over in bed, or straightening up after bending over. BPPV is the most common cause of recurrent vertigo, with a lifetime prevalence of $2.4 \%{ }^{13}$ $\mathrm{BPPV}$ is predominant in the age range between 50 and 55 years ${ }^{14}$ but can reach a cumulative incidence of $10 \%$ in elderly people ( 80 years old). Younger people may develop BPPV as a consequence of head trauma. Women are affected twice as often as men. ${ }^{15}$ It is rare in childhood. BPPV is a form of positional vertigo. Positional vertigo is defined as a spinning sensation produced by changes in head position relative to gravity.

Table 1. Type of dizziness.

\section{Type of dizziness}

\begin{tabular}{lc}
\hline Vertigo & Illusion of movement usually rotatory of self or surrounding \\
\hline Presyncope & Sensation of impending loss of consciousness \\
\hline Disequilibrium & Postural unsteadiness imbalance \\
\hline Lightheadedness & Vague symptoms, possibly feeling disconnected with the environment
\end{tabular}

Table 2. Causes of vertigo.

\begin{tabular}{lc}
\hline Peripheral vertigo (labyrinth, vestibular nerves) & Cause of vertigo \\
\hline Common disease & Central vertigo (central nervous system) \\
\hline Acute vestibulopathy: vestibular neuritis or labyrinthitis & TIA or stroke especially if vertebrobasilar system affected \\
\hline Ménière's disease & Migrainous vertigo \\
\hline Benign positional paroxysmal vertigo & Multiple sclerosis \\
\hline Perilymphatic fistula & Cerebellopontine angle tumor \\
\hline Cholesteatoma erosion & \\
\hline Herpes zoster oticus & \\
\hline Otosclerosis & \\
\hline TIA, transient ischemic attack. &
\end{tabular}


Traditionally, the terms benign and paroxysmal have been used to characterize this particular form of positional vertigo. The term benign implies that BPPV is not due to any serious CNS disorder; the term paroxysmal describes the rapid and sudden onset of the vertigo. However, undiagnosed and untreated BPPV may not have benign functional, health, and quality-of-life impacts. ${ }^{16}$ A quick clinical diagnosis of BPPV can avoid expensive and unnecessary examinations, and lead to a rapid, easy, and effective treatment in $>90 \%$ of cases. ${ }^{15}$ Most BPPV is idiopathic. Causes can be attributed to about $40 \%$ and include: i) head injury; ii) spontaneous degeneration of the labyrinth; iii) post-viral illness, ${ }^{17} \mathrm{iv}$ ) chronic middle-ear disease.

Some authors assume that also a vascular, toxic, idrophic and dismetabolic causes are possible. ${ }^{17}$

\section{Classification}

The classification of BPPV can be based on the anatomical location or the etiology. The most commonly involved semicircular canal is posterior (85$95 \%$ ), occasionally lateral canal and rarely the superior canal. In some cases it may even involve more than one canal at a time $(0.4 \%){ }^{18}$ Posterior semicircular canal BPPV is diagnosed when patients report a history of vertigo provoked by changes in head position relative to gravity and when, on physical examination, characteristic nystagmus is provoked by the Dix-Hallpike maneuver.

\section{Pathogenesis}

BPPV results from abnormal stimulation of the cupula within any of the three semicircular canals. Two main theories, namely cupulolithiasis and canalolithiasis have been propounded over time in an attempt to explain the pathogenesis of BPPV. ${ }^{9}$ Cupulolithiasis theory opined that the degeneration of the utricular otolithic membrane occurs following trauma, ischemia, infections, and other insults of the vestibular organ which results in the release of otoconia which gets deposited at the posterior semicircular (PSSC). This alters the specific gravity of cupula thus converting it to a gravity receptor and thereby provoking a rotatory nystagmus on rapid change of the head position. Canalolithiasis theory proposes that otoconial debris floats freely in the PSSC rather than getting attached to the cupula. Rapid changes in the head position causes downwards movement of this otoconial clots of debris and induces endolymphatic flow and cupular deflection resulting in the typical features of BPPV. The time lag in the aggregation of the otoconial floaters into clots could account for the latent period elicited in BPPV. Currently, it is believed that canalolithiasis theory explains most of the classical features of BPPV and coexists with cupulolithiasis. ${ }^{13}$ Therefore, both theories are thought to be relevant in the pathogenesis of BPPV.

\section{Diagnosis}

Diagnosis of BPPV rests on recognition of characteristic positioning nystagmus in a patient with a typical history of positional vertigo. The Dix Hallpike maneuver is the test most commonly used to induce signs and symptoms of BPPV, and is regarded as the gold standard and critical for accurate diagnosis. The Dix-Hallpike maneuver is performed by the clinician moving the patient through a set of specified head-positioning maneuvers to elicit the expected characteristic nystagmus of posterior canal BPPV. ${ }^{19}$ The patient begins in the seated position with the examiner standing behind the patient, but offset to the side of the ear to be tested. The examiner then gently turns the patient's head 45 degrees to the side to be examined by placing their hands on each side of the patient's face. This rotation aligns the tested posterior semicircular canal with the patient's sagittal plane. Next, while keeping the head rotated 45 degrees, the examiner moves the patient in a rapid but controlled manner to the supine position with the tested ear down, and then gently extends the patient's head beyond the edge of

Table 3. Peripheral and central vertigo.

\begin{tabular}{lcc}
\hline \multicolumn{1}{l}{ Peripheral and central vertigo } & \\
\hline Characteristic & Peripheral & Central \\
\hline Severity & Severe & Mild \\
\hline Onset & Sudden & Gradual \\
\hline Duration & Seconds to minutes & Weeks to months \\
\hline Positional & Yes & No \\
\hline Fatigable & Yes & No \\
\hline Associated symptoms & Auditory & Neurologic and visual \\
\hline Associated nystagmus & Horizontal & Vertical \\
\hline
\end{tabular}


the bed. During the entire maneuver, the patient's eyes remained opened and fixed on the examiner's nose or forehead, while the examiner observes for the latency, direction, and duration of any induced nystagmus. The patient is then returned to the seated position. And after a brief rest, the maneuver is repeated on the opposite side. The side that elicits the positive test is the affected side. ${ }^{18}$ The negative Dix-Hallpike maneuver does not necessarily rule out a diagnosis of posterior canal BPPV. Because of the lower negative predictive values of the Dix-Hallpike maneuver, it has been suggested that this maneuver may need to be repeated at a separate visit to confirm the diagnosis and avoid a false-negative result.

\section{Treatment}

BPPV is a frequent disease, which is usually resolved spontaneously or by office-based canalith repositioning maneuvers. In some cases $(1.7 \%)$, however, it may also be manifested as a persisting and/or recurrent disabling problem. Two types of maneuver have been found effective for posterior canal BPPV: the canalith repositioning procedure (CRP) - also referred to as the Epley maneuver - and the liberatory maneuver (also called the Semont maneuver). ${ }^{15}$ Treatment with canalith repositioning procedure called CRP was first described by Epley in 1992. ${ }^{19}$ Through a series of head position changes, the CRP moves the canaliths from the posterior semicircular canal to the vestibule, thereby relieving the stimulus from the semicircular canal that had been producing the vertigo in BPPV. CRP is most commonly performed in the outpatient setting by a clinician after confirmation of the diagnosis of posterior canal BPPV. ${ }^{20}$ Patients should be informed that nausea, occasional vomiting, and/or a sense of falling may arise during the CRP. ${ }^{21}$ The Semont's liberatory maneuver for cupulolithiasis is characterized by extremely rapid movement as the patient is moved in a cart-wheeling motion from the dependent Dix-Hallpike position to the opposite side through sitting. The speed of the movement is necessary as both inertia and gravity act to remove any particles adhering to the cupula and to prevent the particles falling back toward the ampulla. The maneuver is aggressive and potentially distressing and harmful to patients, especially those with mobility problems. ${ }^{13}$

\section{Vestibular neuritis/labyrinthitis}

The second most frequent cause of vertigo in general population is APV which is an acute peripheral vestibulopathy and it is thought to result from a viral infection or a reactivation of herpes simplex virus that affects the vestibular ganglion, vestibular nerve, labyrinth, or a combination of these. It commonly follows an episode of flu. The facts that the disorder often has a viral prodrome occurring in epidemics, that it may affect several members of the same family, and that it occurs more commonly in spring and early summer all support a viral cause. ${ }^{22}$ Inflammatory and vascular causes are also described. The symptoms are prolonged continuous vertigo, nausea, vomiting and imbalance and last for days to weeks. Some patients can have residual non-specific dizziness and imbalance that lasts for months. When the labyrinth is affected, auditory symptoms are associated. Diagnosis requires physical examination and caloric testing. ${ }^{23} \mathrm{On}$ the basis of the appearance of the nystagmus, a positive head-thrust test, and a negative neurologic examination, one can usually be confident in the diagnosis of a unilateral peripheral vestibulopathy. Electronystagmography, if available, can document the unilateral vestibular loss (i.e., unilateral caloric hypoexcitability) but is rarely necessary. Currently, viral studies (serologic analysis or cultures) are not recommended, since they cannot prove a causal relation between a viral infection and the vestibular syndrome.

Most patients recover well from vestibular neuritis, even without treatment. Antiemetic and vestibular suppressants are useful acutely but should be withdrawn as soon as possible (preferably within the first few days) because their prolonged use may impede the process of central vestibular compensation. The main classes of drugs used for symptoms of acute vertigo include antihistamines, anticholinergic agents, antidopaminergic agents, and $\gamma$-aminobutyric acid-enhancing (GABAergic) agents. ${ }^{24}$ These drugs do not eliminate but rather reduce the severity of vertiginous symptoms. The response is clearly dose-dependent, so if the initial dose is not effective, higher doses should be tried. A vestibular exercise program typically includes exercises designed to improve ocular stability and balance. ${ }^{24}$ While nystagmus is present, the patient should try to suppress it with fixation in all positions of gaze. As the nystagmus diminishes, eye and- headcoordination exercises can be started (e.g., staring at a visual target while oscillating the head from side to side and up and down). Combined movements of the eyes and head involving jumping quickly back and forth between two widely separated targets are also useful. ${ }^{25}$ Prognosis is favorable, although disorders of moderate intensity may rather often persist, in the form of positional vertigo, sometimes paroxysmal, or disorders of equilibrium on abrupt movement. In evaluating a patient with an acute vestibular syndrome, it is important not to miss a central cause, such as a brainstem or cerebellar stroke or hemorrhage, which could be life-threatening. ${ }^{26}$ Indeed, definitive central signs are not always present and some patients have an acute central vestibular syndrome (vestibular pseudoneuritis), resulting from a posterior fossa stroke. In cases of central vestibular syndrome, further diagnostic [e.g., magnetic resonance imaging (MRI), 
Doppler sonography] and management (e.g., thrombolysis) is warranted.

\section{Ménière disease}

Ménière disease is an inner ear disorder characterized by recurrent episodes of rotational vertigo, most typically associated with fluctuating progressive changes in hearing, typically on low frequencies at early stages, fullness and tinnitus. ${ }^{27}$ It is the most common cause of episodic vertigo combined with fluctuating hearing loss. Some causes have already been identified as most probable: extrinsic factors (inflammation, trauma, otosclerosis, autoimmunity, endocrine disorders, and such) interact with congenital (genetic) and developmental intrinsic factors (primary or secondary, acquired) into a multifactorial inheritance that is, to date, the best explanation for the basis of MD. The natural history is for spontaneous remission in 60 to $80 \%$ of the patients, whilst 10 to $20 \%$ have intractable symptoms. ${ }^{27}$ After tinnitus and fullness of the ear, vertigo may come and go with changes in hearing, occur during or just before attacks, or be constant. In most cases only one ear is involved, but both ears may be affected in about 15 percent of patients. MD typically starts between the ages of 20 and 50 years. Men and women are affected in equal numbers. Endolymphatic hydrops is widely accepted as the pathologic substrate, but not all hydrops seems to be progressive or becomes clinically manifest. Endolymphatic hydrops is the result of a dysfunction in the mechanism of production/absorption of endolymph, which is mainly due to defective absorptive activity of the endolymphatic duct and sac. ${ }^{28}$ The diagnosis of MD is by exclusion, and a careful history is the most important guide to a correct diagnosis. ${ }^{29}$ Criteria used to define MD are: the presence of at least two episodes of vertigo of at least 20 min duration, audiometrically confirmed sensorineural hearing loss on at least one occasion, tinnitus or aural fullness during episodes and exclusion of other possible causes of vertigo. ${ }^{30}$ Its medical treatment is largely empiric and includes vestibular sedatives and anti-emetics, like diazepam and promethazine (during the acute attack). A low salt diet, diuretics (e.g., amiloride), vasodilators and avoidance of caffeine are commonly used for prophylaxis. Systematic reviews have shown that intra-tympanic gentamycin was useful in severe disease. Surgery can be considered when, even after medical therapy, the disease progresses and the symptoms become intractable. In patients with intractable symptoms, endolymphatic sac surgery or ablative surgery (vestibular neurectomy, and labyrinthectomy) can be considered. ${ }^{31}$

\section{Superior semicircular canal dehiscence}

In semicircular canal dehiscence syndrome, the bone overlying the superior aspect of the superior semicircular canal becomes thin or even absent, thereby allowing pressure to be transmitted to the inner ear. Vertigo is provoked by coughing, sneezing, and Valsalva maneuver. Patients may experience nausea and instability during brief episodes of vertigo. ${ }^{32}$ The syndrome appears to be the result of a minute hydraulic phenomenon within the inner ear. When sound vibrations are transmitted from the tympanic membrane by way of the ossicles to the oval window, the stapes acts as a piston, pushing into the perilymph of the inner ear. Fluids are not compressible, and to cause even minute movement of the fluid, there must be a compensatory displacement somewhere in the system that coincides with that of the stapes. In the cochlea, the outward movement of the round window membrane balances the inward movement of the stapes in the oval window. A pressure wave moves through the perilymph of the scala vestibuli and scala tympani to the round window membrane. Normally, the oval and round windows are the only two openings in the hydraulic system of the inner ear. The semicircular canals represent a hydraulically closed system, and there is no substantial movement of fluid in the semicircular canals when the stapes vibrates in response to sound. However, if there is creation of a third window in one of the semicircular canals, the hydraulic purity is corrupted, and movement can occur. As the stapes pushes inward, the covering of this third window pushes outward. This causes slight movement of the perilymph. This movement or pressure wave in the perilymph apparently compresses the endolymph within the membranous semicircular canal. The brain interprets motion of the endolymph as movement of the body, and the patient feels dizzy. ${ }^{33}$ This syndrome would represent one of the few causes of conductive hearing loss that results from an abnormality within the labyrinth in a location more central than the oval window. Therefore, superior semicircular canal dehiscence syndrome might be considered a diagnostic possibility for conductive hearing loss, along with other causes, such as otosclerosis.

\section{Central disorders}

\section{Migraine associated vertigo}

Vestibular migraine (VM) designates recurrent attacks of vertigo that are caused by migraine. VM affects more than $1 \%$ of the general population, about $10 \%$ of patients in dizziness clinics and at least $9 \%$ of patients in migraine clinics. It is the main disorder capable of mimicking MD in its early stages and various studies have focused on the higher prevalence of migraine in MD patients, variously reported between $43 \%$ and $56 \%$, while in the normal population it is $10 \% \cdot{ }^{34}$ Clinically, $\mathrm{VM}$ presents with attacks of spontaneous or positional 
vertigo lasting seconds to days. Episodes of vertigo occur in about one fourth of patients and, in some patients, vertigo is the only symptom (so-called migraine equivalent). Migrainous accompaniments such as headache, phonophobia, photophobia or auras are common but not mandatory. Cochlear symptoms may be associated but are mostly mild and non-progressive. During acute attacks one may find central spontaneous or positional nystagmus and, less commonly, unilateral vestibular hypofunction.

\section{Stroke and vertebrobasilar insufficiency}

The most worrisome central cause of dizziness is acute ischemic event in the posterior fossa..$^{35}$ The symptoms and signs vary depending on the location of the ischemic event. Vertigo is among the initial symptoms in $48 \%$ of patients with stroke but stroke is diagnosed in less than $5 \%$ of patients presenting with dizziness. A small percentage of patients with vertebral basilar ischemia may present with isolated spells of vertigo, presumably due to ischemia in the distribution of the anterior vestibular artery, a branch from the anterior inferior cerebellar artery (AICA). This small artery perfuses the anterior and lateral SCC and the utricul maculae, and spares the cochlea. As far as the clinical examination is concerned the vestibule-ocular reflex (VOR) is normal unless the peripheral vestibular system is involved (infarct in the dorsolateral pons-AICA). Selective lesions in the central vestibular pathways result in spontaneous nystagmus: unlike spontaneous nystagmus from peripheral vestibular lesions, those from central vestibular lesions are not readily suppressed with fixation. ${ }^{36} \mathrm{~A}$ positive Romberg test may be found in patients with proprioceptive defects from dorsal root ganglia or dorsal column disease. Gait is a very helpful test to assess patients with central defect because it is often impaired even when few additional findings are noted. The gait may be spastic, ataxic, halting, or freezing. The central vestibular structures are perfused by several vessels within the vertebral basilar system with subsequent different signs and symptoms. ${ }^{36}$ There are only anecdotal comments about the effectiveness of vertigo treatment from cerebrovascular disorders. Ondansetron may be appropriate for severe vertigo and nausea from stroke. Coumadin and transluminal angioplasty of vertebral artery stenosis and, occasionally, aspirin and ticlopidine have been found to be effective in stopping spells of central vertigo from vertebrobasilar artery insufficiency. Treatment includes reduction of risk factors for cerebrovascular disease and antiplatelet therapy.

\section{Transient ischemic attacks}

Transient ischemic attacks (TIA) from vertebrobasilar ischemia provoke episodes of dizziness that are abrupt and usually last for only a few minutes. TIA frequently are associated with other vertebral basilar insufficiency (VBI) symptoms, most commonly visual disturbance, drop attacks, unsteadiness or weakness. A small percentage of patients with VBI may present with isolated spells of vertigo, presumably caused by ischemia in the distribution of the vestibular artery. These patients usually have known cerebrovascular disease or risk factors for this disease. MR arteriography can be performed to assess the vessels of the posterior circulation, and transcranial Doppler may detect decreased flow in the basilar artery. Treatment includes reduction of risk factors for cerebrovascular disease and antiplatelet therapy. ${ }^{35}$

\section{Demyelinating disease}

Multiple sclerosis, the most common demyelinating disorder, is frequently associated with dizziness. ${ }^{37}$ In approximately one out of five patients with multiple sclerosis the initial presentation will be disturbances of balance and gait. Some patients with multiple sclerosis who present with vestibular symptoms do so because of the involvement of the root entry zone of the VIII cranial nerve. Also, these patients often manifest an internuclear ophthalmoplegia. They can experience symptoms of dizziness and disequilibrium on the basis of blurred vision and transient diplopia. A diagnosis of multiple sclerosis requires the identification of fluctuating neurologic symptoms, which can present in different time. Magnetic resonance imaging of the brain can be most helpful in establishing diagnosis of multiple sclerosis. Although most dizzy patients with multiple sclerosis manifest multiple signs and symptoms referable to various locations in the nervous system, isolated involvement of the root entry zone of the vestibular portion of the VIII nerve can present in a manner virtually indistinguishable from that of vestibular neuritis. $^{38}$

\section{Mass lesions}

Acoustic neuroma (best called vestibular schwannoma) and other cerebellopontine angle mass are considered in the category of mixed disorders because the involvement of dura, brainstem, cerebellum, or other cranial nerves can produce other neurologic findings in addition to the peripheral vestibular and cochlear ones. ${ }^{39}$ Moreover, the effects of brainstem or cerebellar compression can produce central vestibular signs that occur with or in place of peripheral ones. The patient with a cerebellopontine angle tumor may report balance symptoms that reflect the anatomic extent of the tumor. The classic clinical manifestation of a small acoustic neurinoma is initial unilateral tinnitus and mild unilateral hearing loss. As the tumor grows the patients will note progressive hearing loss in the involved ear and may experience vertigo. The vertigo, 
however, is usually short lived and eventually resolves or is replaced by a much milder disequilibrium. As the tumor becomes large, the trigeminal nerve may become compressed with variable symptoms, numbness, facial pain, and trigeminal neuralgia. As the brainstem becomes compressed, hydrocephalus ensues with associated headaches and visual loss. If untreated, persistent growth results in lower cranial nerve dysfunction, ataxia, hemiparesis, and respiratory arrest. Early symptoms include hearing loss, tinnitus, and disequilibrium. Late symptoms include these as well as headache, trigeminal symptoms, and hydrocephalus. $^{40}$

\section{Orthostatic hypotension}

Symptoms of orthostatic hypotension can range from lightheadedness when first standing up to chronic tiredness, mental slowing, dizziness, nausea and impending syncope. ${ }^{41}$ Common causes include medications (diuretics, antihypertensive medications, tricyclic antidepressants), prolonged bed-rest, and neurogenic causes (autonomic neuropathy from diabetes, amyloidosis, multiple system atrophy, Parkinson's disease). Diagnosis is made on the basis of a drop in systolic pressure by $20 \mathrm{mmHg}$ or more when the patients stands associated with symptoms of lightheadedness. Management begins with the removal of potentially offending drugs if possible and rehydration if necessary. Salt and fluid intake should be increased. ${ }^{42}$

\section{Panic attacks}

A panic attack is an anxiety disorder that causes intense fear or discomfort that reaches a crescendo within $10 \mathrm{~min}$; panic attacks are frequently associated with dizziness, nausea, shortness of breath or chest tightness, paresthesia and sweating. An attack may occur unexpectedly or be situational bound. ${ }^{43}$ Chronic anxiety may also present with dizziness. The selective serotonin release-uptake inhibitors have been approved for panic disorder. Alprazolam can be used sporadically if attacks are infrequent. ${ }^{44}$

\section{Cervical vertigo}

Central nervous system trauma can produce dizziness especially as a feature of so-called post-concussion syndrome..$^{45}$ This dizziness is often nonspecific, and its origin is uncertain. Possibly, a brainstem rather than a cerebral concussion is responsible for dizziness in some patients. The role of the neck in balance cannot be overlooked. Although the diagnostic entity of cervical vertigo is poorly described and nearly impossible to diagnose definitively, the central nervous system must recognize the position of the head with respect to the torso for normal vestibule-spinal func- tion. Any traumatic injury that impairs the ability of central nervous system to locate accurately the head on the torso, for example a neck injury, can produce dizziness and disequilibrium. Often people complain of dizziness after a whiplash injury of the neck. They may complain of a swimming sensation in the head, or headache, and often have restrictions in cervical range of motion. These patients may have referred pain in their shoulder and scapular region and may have difficulty in sleeping because of neck pain. The diagnosis of cervical vertigo usually represents a diagnosis of exclusion. ${ }^{46}$

\section{The management of patient with vertigo: rationale and objective}

Vertigo is a symptom, not a disease and effective diagnosis and management begin with understanding what this symptom may represent. Vertigo is a common symptom that can depend on peripheral and central causes and affects $20 \%$ to $30 \%$ of the general population. For these reasons the diagnosis and management of vertigo should be also a competence of internist. Therefore, the goal of this work is to raise awareness of the clinical management of vertigo through a better knowledge of its diagnosis and its treatment.

\section{The management of vertigo: methodology}

In order to provide evidence-based recommendations for the management of patients with vertigo, we first verified the existence of guidelines on the matter. Therefore, we conducted a search using the following database guidelines:

- Scottish Intercollegiate Guidelines Network (SIGN); - Institute for Clinical System Improvement (ICSI);

- National Institute for Health and Clinical Excellence

(NICE) (NHS evidence);

- National Guideline Cleringhouse;

- Canadian Medical Association, CMA Infobase;

- New Zeland Guidelines Group;

- National Sistem Guidelines;

- Clinical Practice Guidelines Portal;

- eGuidelines.

The research was carried out by five authors independently, using as key-words terms vertigo, dizziness, when the site included the search function, and in other cases we listed the last manually guidelines stored in the database or made reference to the neurological and otological illness. The results obtained separately were then compared and discussed together. The guidelines thus obtained were evaluated using the AGREE instrument (Appraisal of Guidelines, Research and Evaluation II, 22) ${ }^{47}$ by 4 authors independently. AGREE II assesses compliance with 23 
requirements, meeting 6 domains as the explanation of the purpose, the clarity, the involvement of all stakeholders, the rigor of development, applicability and editorial independence of the same. Each author assessed the compliance of individual requirements with a score from 1 (disagree completely) to 7 (complete agreement). The scores assigned by each author were added within individual domains and reported with the highest and the lowest score possible within the domain based on the number of requirements included and the number of evaluators.

\section{The management of vertigo: results}

Through the databases listed above, we identified 46 guidelines: 39 were excluded based on the title or the text or for other reasons and 7 were selected for evaluation. The two guidelines derived from the New Zealand Guidelines Group ${ }^{48,49}$ were really signs for the patient suffering from vertigo and his family and were therefore excluded. The guideline produced by NICE ${ }^{50}$ was focused on micropressure therapy for MD and thus too specific and non-functional for our work. The guidelines produced by the Clinical Practice guidelines ${ }^{11,51}$ even if they are a valid tool for the diagnosis and management of vertigo are not really strictly guidelines. The two guidelines derived from National Guideline Cleringhouse ${ }^{52,53}$ were evaluated. The first guideline (ACR appropriateness criteria vertigo and hearing loss $)^{52}$ has the goal of evaluating the appropriateness of initial radiological examinations for patients with vertigo and hearing loss and therefore focused only on a single specific aspect of the management of the patient with vertigo (score 3.5). The second guideline (Clinical practice guideline: benign paroxysmal positional vertigo $)^{53}$ is a good guideline (score 5) although only focused on one of the diseases that can cause vertigo. For this reason the evidencebased medicine management of vertigo was obtained also by the analysis of reviews and articles on vertigo and dizziness. ${ }^{1,7-9,54-58}$

\section{Clinical approach to patients with vertigo}

Patients with dizziness have to be assessed for the type of sensitive symptoms. Then the physician, through medical history and physical examination, differentiates vertigo from other conditions like peripheral neuropathy, Parkinson's disease, heart disease or anxiety.

\section{History}

The history is the most important part of the evaluation. When a patient presents with vertigo or dizziness, the first step is to delineate more precisely the nature of the symptoms. ${ }^{11,54}$ Table $3^{11}$ shows the characteristics that distinguish central from peripheral vertigo. Then, a careful history can differentiate a vague dizziness in disequilibrium, primarily a balance problem, from the subjective sensation of dizziness in the head and in this case we have to determine if the sensation is lightheadedness or vertigo. Symmetric bilateral vestibular hypo-function causes imbalance but no vertigo. The history should focus closely on other features, including whether dizziness is paroxysmal or occurred only once, the duration of each episode, any provoking factors, and the symptoms that accompany the dizziness. The anamnestic history is also relevant. The recent upper respiratory tract infection or ear infection, migraine, head trauma or recent labyrinthitis, direct, anxiety or depression, drugs, acute intoxication with alcohol, family history of migraine or Ménière's can be found. Because of the ambiguity in patients' descriptions of their symptoms, diagnosis based simply on symptom character is typically unreliable. There are three main characters in the history of dizziness to evaluate: the time, the symptoms and the circumstances. On the basis of time, symptoms, circumstance it is possible to distinguish between the various causes of headache.

\section{The time}

Causes of dizziness can be divided into episodes that last for seconds, minutes, hours, or days. Therefore it needs to evaluate the characteristic of attack and the spells of dizziness. The acute attack of dizziness is 3 days or less while the chronic dizziness is more than three days. Common causes of brief dizziness (seconds) include BPPV and orthostatic hypotension, both of which are typically provoked by changes in position. Attacks of migraines vertigo and MD often last hours. When episodes are of intermediate duration (minutes), transient ischemic attacks of the posterior circulation should be considered, although these episodes could be also due to migraine or a number of other causes. ${ }^{2,11}$

\section{The symptoms}

It is important to ask the patient about associated symptoms. The patient can have otological symptoms (hearing loss, discharge, tinnitus), or/and neurological symptoms (headache, diplopia, visual disturbance, dysarthria or dysphagia, paresthesia, ataxia) or/and general autonomic symptoms (nausea and vomiting, sweating, palpitations) or/and migraine aura symptoms (visual or olfactory symptoms). In the case of vestibular disorders, the physical symptoms depend on whether the lesion is unilateral or bilateral and whether it is acute or chronic and progressive. Unilateral hearing loss and other aural symptoms (ear pain, pressure, fullness) typically point to a peripheral cause. Because the auditory 
pathways quickly become bilateral upon entering the brainstem, central lesions are unlikely to cause unilateral hearing loss (unless the lesion lies near the root entry zone of the auditory nerve). Symptoms such as double vision, numbness, and limb ataxia suggest a brainstem or cerebella lesion. ${ }^{2}$

\section{The circumstances}

Knowledge of the circumstances triggering or exacerbating symptoms offer helps to elucidate the mechanism behind dizziness. If it is reported that movements exacerbate symptoms. Thus it is important to differentiate whether the movements are primarily head movements, body movements, postural changes, or eye movements. Dizziness may be provoked by only certain movements, such as standing up after lying down for at least 10 ' in orthostatic hypotension, or may occur after vertical or oblique head movements, such as lying down, turning over in bed, or sitting up in BPPV. If the individual tells you that simply moving the eyes with the head stationary causes dizziness and there is no eye movement disorder (ocular misalignment or an internuclear ophthalmoparesis), then dizziness is likely to be the result of anxiety. ${ }^{54,55}$

\section{Examination}

The five key elements in the clinical examination of a dizzy patient are assessment: VOR, spontaneous nystagmus, positional nystagmus, the Romberg test and gait.

\section{Spontaneous nystagmus}

Nystagmus is a term used to describe alternating slow and fast movements of the eyes. Patients with vestibular neuritis have a peripheral vestibular pattern of nystagmus. In this setting, the peripheral vestibular pattern is a unidirectional, principally horizontal pattern of nystagmus. This description means that the nystagmus beats in only one direction. Conversely, bidirectional gaze-evoked nystagmus is a central nervous system pattern of nystagmus. Other central nervous system patterns are pure torsional nystagmus or spontaneous vertical (typically down-beat) nystagmus. With an acute peripheral vestibular lesion, the only pattern of nystagmus that can result is unidirectional nystagmus. In acute severe dizziness presentation, any other pattern should be considered a central nervous system sign. When an acute lesion occurs on one side, the input from the opposite side is unopposed. As a result, the eyes will be pushed toward the lesion side. This movement of the eyes is the slow phase of the nystagmus. When the eyes reach a critical point off center, the brain responds by generating a corrective eye movement to move the eyes back. This is the fast phase of nystagmus. ${ }^{24}$

\section{Vestibule-ocular reflex}

A recently described bedside test, the head-thrust test, is now an important component of the bedside evaluation in acute severe dizziness presentation..$^{57,58}$ The test allows the examiner to assess VOR on each side. The VOR is the component of the vestibular system that triggers eye movement in response to stimulation. The intact VOR compensates for head movements by moving the eyes in the opposite direction to head movements. To test the VOR using the head thrust test, the examiner stands in front of the patient and grasps the patient's head with both hands. The patient is instructed to focus on the examiner's nose and then the examiner initiates a quick 5 to 10 degree movement of the patient's head to one side. When there is a lesion of the VOR on one side, as occurs with vestibular neuritis, a corrective eye movement (saccade) back to the examiner's nose is seen after the head is moved toward the affected side. In contrast, serving as internal control, the eyes will stay on target after the head thrust toward the normal side because the VOR is intact on that side. The reason for the corrective saccade with a peripheral vestibular lesion is rooted in the physiology of the vestibular system. When the head is moved quickly in one direction, the reflex that moves the eyes toward the opposite direction is generated by the side the head moved toward. Thus a patient with vestibular neuritis of the left side will present with spontaneous right beating unidirectional nystagmus and have a positive head thrust test with movements toward the left side..$^{25,59}$

\section{Positional nystagmus}

Positional nystagmus and vertigo are best assessed during the Dix-Hallpike test. ${ }^{59}$ Normally there is no nystagmus or vertigo with this maneuver. A positive Dix-Hallpike test will result in transient vertigo and torsional nystagmus, after a 30 " delay, with the head turned towards the affected ear.

\section{Romberg test}

In the Romberg test the patient is asked to stand with feet slightly apart, first with eyes open, and then closed. Then the patient has to fold his arms across the chest for 30 " eyes open and then 30 " eyes closed. Romberg test is positive if the patient is stable with eyes open but loses balance with eyes closed.

\section{Gait}

The patient should be asked to walk for about 20 $\mathrm{ft}$ in one direction and then to turn and come back. Features to look for are gait speed, the base width, step length, arm swing, deviation of path and movement soft the head. 


\section{The management of vertigo}

The management of vertigo consisted of three steps: i) quickly identify if the problem is peripheral (inner ear or vestibular nerve); ii) determine if the patient needs to be admitted to the hospital or referred to a specialist clinic; iii) offer initial management of the problem. ${ }^{12}$

\section{Quickly identify if the problem is peripheral (inner ear or vestibular nerve)}

On the basis of some characteristics (severity, onset, duration, positional, fatigable, symptoms associated and type of nystagmus) it is possible to distinguish between central or peripheral causes (Tables 2 and 3). ${ }^{11,12}$

\section{Determine if the patient needs to be admitted to the hospital or referred to a specialist clinic}

The major causes of vertigo are not a serious health threat and many cases of vertigo will resolve on their own. When a central cause for vertigo is suspected based on the history and clinical examination imaging should be carried out. ${ }^{11}$ Only the patient with extreme dehydration (from vomiting) or if central disorder is suspected should be admitted to the hospital.

\section{Offer initial management of the problem}

An acute and severe episode of vertigo, regardless of the underlying cause, will usually settle by itself within 24-48 h due to the effect of brainstem compensation. During the acute phase, supportive measures, bed rest, antiemetics and vestibular blocking agents can be used to provide symptomatic relief. As soon as the cause of vertigo has been found (Figure 1), ${ }^{1}$ the patient should receive treatment for the disease that caused the symptom of vertigo (Tables 4 and 5)..$^{51,60}$

\section{Conclusions}

The vertigo is not just fear of falling but a symptom caused by various diseases. First, it is important

Table 4. Treatment for acute vertigo.

\begin{tabular}{lcc}
\hline Class of drug & Drug & Dosage \\
\hline Antihistamines & Promethazine & $12.5-25 \mathrm{mg}$ orally im or rectally every $4-12 \mathrm{~h}$ \\
\hline Benzodiazepines & Behatistine & $4-6 \mathrm{mg} 8 \mathrm{~h}$ per day \\
\hline & Diazepam & $2-10 \mathrm{mg}$ orally or iv every $4-8 \mathrm{~h}$ \\
\hline Antiemetics & Lorazepam & $0.5-2 \mathrm{mg}$ orally, im or ev every $4-8 \mathrm{~h}$ \\
\hline & Prochlorperazine & $5-10 \mathrm{mg}$ orally or im every $6-8 \mathrm{~h}$ or $25 \mathrm{mg}$ rectally every $12 \mathrm{~h}$ \\
\hline
\end{tabular}

im, intramuscularly; iv, intravenously; ev, endovenously.

Table 5. Treatment of causes of vertigo.

\begin{tabular}{lc}
\hline Cause & $\begin{array}{c}\text { Treatment } \\
\text { Benign paroxysmal positional vertigo }\end{array}$ \\
\hline Ménière disease & $\begin{array}{c}\text { Meclizine } 25-50 \text { mg orally every } 4 \text { to } 6 \mathrm{~h} \\
\text { Epley maneuver } \\
\text { Vestibular rehabilitation }\end{array}$ \\
\hline Vestibular neuritis & $\begin{array}{c}\text { Salt restriction ( }<1-2 \text { g to sodium per day) and/or diuretics } \\
\text { Intratympanic dexamethasone or gentamicin } \\
\text { Endolymphatic sac surgery }\end{array}$ \\
\hline Migrainous vertigo & Methylprednisolone 100 mg orally daily then tapered to 10 mg orally daily over three weeks \\
\hline
\end{tabular}




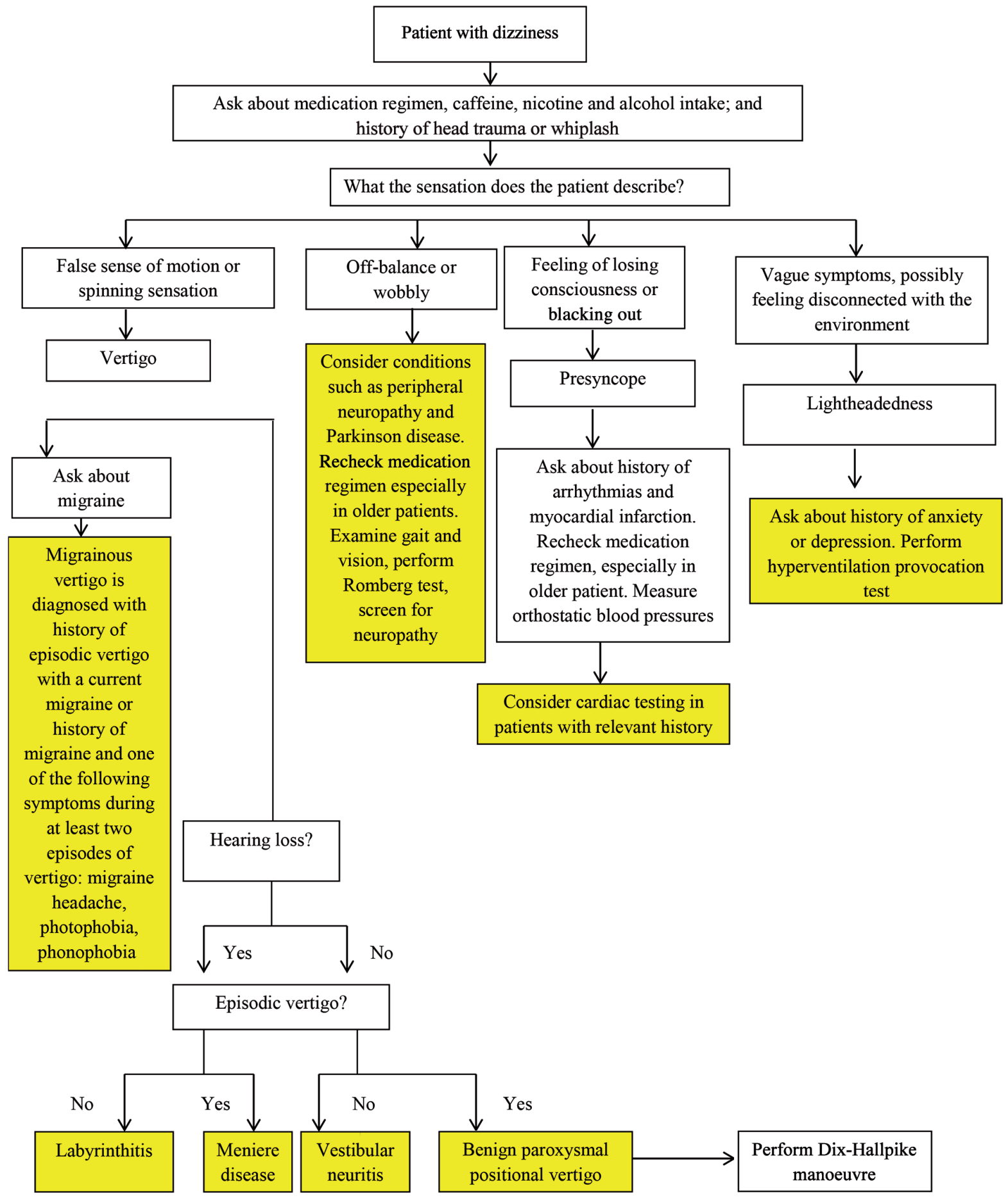

Figure 1. Approach to patient with dizziness.

to distinguish between the various causes of dizziness but above all to recognize the causes that need a prompt treatment. Furthermore vertigo is a common disease and as patients age vertigo increases. For this reason it is important that the internists know diagnose, manage and treat this symptom and then the vertigo may be his/her competence.

\section{References}

1. Post R, Dickerson L. Dizziness: a diagnostic approach. Am Fam Physician 2000;82: 361-8, 369.

2. Chawla N, Olshaker JS. Diagnosis and management of dizziness and vertigo. Med Clin North Am 2006;90: 291-304. 
3. Karatas M. Central vertigo and dizziness: epidemiology, differential diagnosis, and common causes. Neurologist 2008;14:355-64.

4. Neuhauser HK. Burden of dizziness and vertigo in the community. Arch Internal Med 2009;169:89.

5. Newman HK, Toker DE, Cannon LM, et al. Imprecisation in patient reports of dizziness symptom quality: a cross-sectional study conducted in an acute care settings. Mayo Clin Proc 2007:82:1329-40.

6. Skoien AK, Wilhemsen K, Gjesdal S. Occupational disability caused by dizziness and vertigo: a register-based prospective study. Br J Gen Pra 2008;58;619-23.

7. Madlon Kay DJ. Evaluation and outcome of the dizzy patient. J Fam Pract 1985;21:109-13.

8. Herr RD, Zun L, Mathews JJ. A directed approach to the dizzy patient. Ann Emerg Med 1989;18:664-72.

9. Roberts DS, Lin HW, Bhattacharyya N. Health care practice patterns for balance disorders in the elderly. Laryngoscope 2013;123:2539-43.

10. Furman JM, Cass SP. A practical work-up for vertigo. Contemp Intern Med 1995;7:24-7.

11. Kuo CH, Pang L, Chang R. Vertigo - part 2 - management in general practice. Austr Fam Physician 2008;37: 409-13.

12. Tusa RJ, Gore R. Dizziness and vertigo: emergencies and management. Neurol Clin 2012;30:61-74.

13. Von Brevern M, Radtke A, Lezius F, et al. Epidemiology of benign paroxysmal positional vertigo: a population based study. J Neurol Neurosurg Psychiatr 2007; 78:710-5

14. Baloh RW. Vertigo. Lancet 1998;352:1841-6.

15. Barber HR, Leigh RJ. Benign postural vertigo: diagnosis and treatment. In: Barber MO, Sharpe JA, eds. Vestibular disorder. Chicago: Mosby Year Book Medical Publishers; 1988. pp 201-12.

16. Kerber KA. Benign paroxysmal positional vertigo: opportunities squandered. Ann N Y Acad Sci 2015 [Epub ahead of print].

17. Gacek RR. Evidence for a viral neuropathy in recurrent vertigo. ORL J Otorhinolaryngol Relat Spec 2008;70:6-14.

18. Xiang-Dong G. Benign paroxysmal positional vertigo. J Neurosci Rural Pract 2011;2:109-10.

19. Epley JM. The canalith repositioning procedure: for treatment of benign paroxysmal positional vertigo. Otolaryngol Head Neck Surg 1992;107:399-404.

20. Fife TD, Iverson DJ, Lempert T. Practice parameter: therapies for benign paroxysmal positional vertigo (an evidence-based review). Report of the quality. Standards Subcommittee of the American Academy of Neurology. Neurology 2008;70;2067.

21. Uneri A. Falling sensation in patients who undergo the Epley maneuver: a retrospective study. Ear Nose Throat J 2005;84:82, 84-5.

22. Davis LE. Comparative experimental viral neurolabyrinthitis. Am J Otolaryngol 1990;11:382-8.

23. Sloane PD, Baloh RW, Honrubia V. The vestibular system in the elderly: clinical implications. Am J Otolaryngol 1989;10:422-9.

24. Baloh RW, Honrubia V. Clinical neurophysiology of the vestibular system. Contemp Neurol Ser 1979; 18:1-21.

25. Kattah IC, Talked AV, Wang DZ, et al. Hints to diagnose stroke in the acute vestibular syndrome: three-step bedside oculomotor examination more sensitive than early MRI diffusion-weighted imaging. Stroke 2009;40:3504-10.

26. Walker M. Treatment of vestibular neuritis. Curr Treat Options Neurol 2009;11:41-5.

27. Gordon AG. Ménière's disease. Lancet 2006;367:984.

28. James A, Thorp MA. Menière's disease. Clin Evid 2003; 116:565-73.

29. Mancini F, Catalani M, Carru M, et al. History of Ménière's disease and its clinical presentation. Otolaryngol Clin North Am 2002;35:565-80.

30. Da Costa SS, De Sousa LC, Piza MR. Ménière's disease: overview, epidemiology, and natural history. Otolaryngol Clin North Am 2002;35:455-95.

31. Committee on Hearing and Equilibrium. Guidelines for the diagnosis and evaluation of therapy in Ménière's disease. Otolaryngol Head Neck Surg 1995;113:181-5.

32. Minor LB. Superior canal dehiscence syndrome. Am J Otol 2000;21:9-19.

33. Minor LB, Cremer PD, Carey JP, et al. Symptoms and signs in superior canal dehiscence syndrome. Ann N Y Acad Sci 2001;942:259-73.

34. Lempert T, Neuhauser H. Epidemiology of vertigo, migraine and vestibular migraine. J Neurol 2009;256:333-8.

35. Grad A, Baloh RW. Vertigo of vascular origin. Arch Neurol 1989;46:281-4.

36. Gomez CR, Cruz-Flores S, Malkoff S. Isolated vertigo as a manifestation of vertebro-basilar ischemia. Neurology 1996;47:94-7.

37. Pula JH, Newman-Toker DE, Kattah JC. Multiple sclerosis as a cause of the acute vestibular syndrome. J Neurol 2013;260:1649-54.

38. Peyvandi A, Naghibzadeh B, Ahmady Roozbahany N. Neuro-otologic manifestations of multiple sclerosis. Arch Iran Med 2010;13:188-92.

39. Kharkheli ESH, Shurygina LS, Davitashvili OZ, et al. Acoustic neuroma diagnosis. Georgian Med News 2011;192:21-8.

40. Myrseth E, Møller P, Wentzel-Larsen T, et al. Untreated vestibular schwannomas: vertigo is a powerful predictor for health-related quality of life. Neurosurgery 2006; 59:67-76.

41. Ferrer-Gila T, Rizea C. Orthostatic hypotension in the elderly. Rev Neurol 2013;56:337-43.

42. Mager DR. Orthostatic hypotension: pathophysiology, problems, and prevention. Home Health Nurse 2012; 30:525-30.

43. Lempert T, Brandt T, Dieterich M, et al. How to identify psychogenic disorders of stance and gait. J Neurol 1991;238:140-6.

44. Fava GA, Zieezny M, Savron G. Long-term effects of behavioural treatment for panic disorder with agoraphobia. Br J Psychiatry 1995;166:87-92.

45. Friedman JM. Post-traumatic vertigo. Med Health R I 2004;87:296-300.

46. Marzo SJ, Leonetti JP, Raffin MJ, et al. Diagnosis and management of post-traumatic vertigo. Laryngoscope 2004; 114:1720-3.

47. AGREE Next Steps Consortium. Appraisal of Guidelines for Research \& Evaluation II - AGREE II Instrument; May 2009. Available from: http://www.agreetrust. org/wp-content/uploads/2013/10/AGREE-II-Users-Manual-and-23-item-Instrument_2009_UPDATE_2013.pdf 
48. Ministry of Health - Manatū Hauora. Vertigo. Available from: http://www.health.govt.nz/your-health/conditionsand-treatments/diseases-and-illnesses/vertigo

49. Ministry of Health - Manatū Hauora. Dizziness. Available from: http://www.health.govt.nz/your-health/conditions-and-treatments/diseases-and-illnesses/dizziness

50. National Institute for Health and Care Excellence (NICE). Micropressure therapy for refractory Ménière's disease. NICE interventional procedure guidance [IPG426]; April 2012. Available from: http://www.nice. org.uk/guidance/IPG426

51. Kuo CH, Pang L, Chang R. Vertigo - part 1 - assessment in general practice. Austr Fam Physician 2008;37:341-7.

52. Angtuaco EJ, Wippold FJ, Cornelius RS, et al. ACR Appropriateness Criteria ${ }^{\circledR}$ hearing loss and/or vertigo. Reston (VA): American College of Radiology (ACR); 2013. Available from: http://www.guideline.gov/content. aspx?id=47674
53. Bhattacharyya N, Baugh RF. Clinical practice guideline: benign paroxysmal positional vertigo. Otolaryngol Head Neck Surg 2008;139:S47-81.

54. Wetmore SJ, Eibling DE, Goebel JA, et al. Challenges and opportunities in managing the dizzy older adult. Otolaryngol Head Neck Surg 2011;144:651-6.

55. Della-Morte D, Rundek T. Dizziness and vertigo. Front Neurol Neurosci 2012;30:22-5.

56. Kerber KA. Vertigo and dizziness in the emergency department. Emerg Med Clin N Am 2009;27:39-50.

57. Halmagyl GM, Curthoys IS. A clinical sign of canal paresis. Arch Neurol 1988;45:737-9.

58. Tusa RJ. Vertigo. Neurol Clin 2001;19:23-55, v.

59. Lewis RF, Carey JP. Images in clinical medicine. Abnormal eye movements associated with unilateral loss of vestibular funxtion. N Engl J Med 2006;35:e26.

60. Swartz R, Longwell P. Treatment of vertigo. Am Fam Physician 2005;71:1115-22. 